Physics

Physics Research Publications

Purdue University

Year 2003

\title{
Lyman spectrum of holes bound to substitutional 3d transition metal ions in \\ a III-V host: $\mathrm{GaAs}(\mathrm{Mn} 2+, \mathrm{Co} 2+$, or $\mathrm{Cu} 2+), \mathrm{GaP}(\mathrm{Mn} 2+)$, and $\operatorname{InP}(\mathrm{Mn} 2+)$
}

E. Tarhan

S. Rodriguez

I. Miotkowski

A. K. Ramdas

This paper is posted at Purdue e-Pubs.

http://docs.lib.purdue.edu/physics_articles/482 


\title{
Lyman spectrum of holes bound to substitutional 3d transition metal ions in a III-V host: $\operatorname{GaAs}\left(\mathrm{Mn}^{2+}, \mathrm{Co}^{2+}\right.$, or $\left.\mathrm{Cu}^{2+}\right), \operatorname{GaP}\left(\mathrm{Mn}^{2+}\right)$, and $\mathrm{InP}\left(\mathrm{Mn}^{2+}\right)$
}

\author{
Enver Tarhan, I. Miotkowski, S. Rodriguez, and A. K. Ramdas
}

Department of Physics, Purdue University, West Lafayette, Indiana 47907

(Received 20 November 2002; published 6 May 2003)

\begin{abstract}
The $3 d$-transition metal impurities $(\mathrm{Mn}, \mathrm{Co}$, and $\mathrm{Cu}$ ) incorporated into III-V zinc blende hosts ( $\mathrm{GaAs}, \mathrm{GaP}$, or InP) exhibit well resolved excitation lines followed by a photoionization continuum in their infrared absorption spectra. They are associated with transitions from a " $1 s$-like" ground state to various " $p$-like" excited states characteristic of a hole bound to a Coulomb center. Their spacings agree remarkably well with those predicted in the effective mass theory for single acceptors [A. Baldereschi and N. O. Lipari, Phys. Rev. B 9, 1525 (1974)] as expected for $3 d$-transition metal ions substitutionally replacing the group III cations of the host. The shape of the photoionization spectra, the occurrence of the simultaneous excitation of the Lyman transitions in combination with the zone center longitudinal optical phonon and hence lying in the photoionization continuum and displaying Fano-like asymmetries, the additional continuum excitations to and beyond the $p_{1 / 2}$ valence band maximum, are the features described and interpreted. $\mathrm{Cu}$ acceptors in GaAs show Lyman doublets, indicative of two independent centers; it is speculated that they could arise from He-like $3 d^{10} 4 s^{2} 4 p^{1}$, $\mathrm{Cu}^{2-}$, and $\mathrm{H}-$ like $3 d^{9} 4 s^{2} 4 p^{1}, \mathrm{Cu}^{-}$, Coulomb centers.
\end{abstract}

DOI: 10.1103/PhysRevB.67.195202

PACS number(s): 75.50.Pp, 78.40.Fy, 78.30.-j

\section{INTRODUCTION}

It is well known that the incorporation of a $3 d$ transition metal ion (TMI) in a II-VI semiconductor by the substitutional replacement of the group II cation results in a remarkable class of II-VI ternary alloys known as a diluted magnetic semiconductor (DMS). ${ }^{1}$ The electronic structure of a free $3 d$ TMI is $[\mathrm{Ar}] 3 d^{n} 4 s^{2}(n=1,2, \ldots, 9)$; when it replaces a group II ion, the $s p^{3}$ tetrahedral bonding scheme takes up the two $4 s$ electrons from the cation and the six $n s^{2} n p^{4}(n=2,3,4$, or 5) from the anion. Thus in a manner similar to that of a group II ion, the substitution of a $3 d$ TMI produces an "isoelectronic" impurity. The remarkable magnetic behavior in the II-VI DMS's is traced to the ground state of the incomplete $3 d$ shell of the TMI subject to crystal field and spin-orbit splittings. The (typically) large magnetic moment of the TMI in the host, the ferromagnetic or antiferromagnetic $d$ - $d$ interactions between them, the spin-spin $s p-d$ exchange interaction between the $3 d$ electrons and the band electrons ( $s$-like conduction band and $p$-like valence band) - these are the microscopic ingredients which generate the spectacular magnetic and magneto-optic phenomena for which the II-VI DMS's are well known.

In the context of the above discussion, it is of great scientific interest to enquire whether corresponding effects could occur in the III-V's when $3 d$ TMI's are incorporated in them. Indeed, this point of view was formulated and implemented by Munekata et $a l^{2}$ more than a decade ago in the growth of $\operatorname{In}_{1-x} \mathrm{Mn}_{x}$ As $(0 \leqslant x \leqslant 0.18)$ exploiting molecular beam epitaxy (MBE). The coexistence of the magnetic moment of the substitutional $3 d$ ion and the presence of holes in the valence band led to the realization that the spin of the charge carrier can be influenced (polarized) in novel device configurations. ${ }^{3-6}$ This new class of semiconductors have been referred to as "spintronic" materials. A striking feature of the III-V spintronic materials is the manner in which free carriers can influence the $d-d$ interaction between the $3 d$ TMI's. ${ }^{6}$

Consider, for example, the introduction of $\mathrm{Mn}$ ions in $\mathrm{GaAs}$ replacing $\mathrm{Ga}$. The cationic substitution can occur with $\mathrm{Mn}$ in one of two electronic configurations (i) $\mathrm{Mn}^{3+}$ in which the requisite three electrons for tetrahedral bonding originate within the new cation (two $4 s^{2}$ and a $d$ electron) or (ii) $\mathrm{Mn}^{2+}$, with the two $4 s^{2}$ electrons provided by the impurity and the third "borrowed" from the valence band of the host. In (i), the TMI is a neutral isoelectronic impurity whereas in (ii), it is an acceptor impurity binding a single hole. The III-V's as diluted magnetic semiconductors can thus occur in two ways, each with a magnetic behavior distinct from that of the other. In case (ii), the $3 d$ shell of the TMI impurity is left intact as it is in the II-VI DMS; however, being an acceptor in the III-V DMS, the acceptorbound hole will display electronic levels not unlike those of a shallow group II impurity, $\mathrm{Zn}$ replacing $\mathrm{Ga}$ in GaAs, for example. A comparison of the Lyman spectra of the shallow acceptors in $\mathrm{GaAs}$, e.g., $\mathrm{Zn}$ replacing $\mathrm{Ga}$ or $\mathrm{C}$ replacing As, on the one hand, and those of the $3 d$ TMI's on the other, is of special interest in the context of the acceptor states in compound semiconductors. Chapman and Hutchinson ${ }^{7}$ more than thirty years ago, and Linnarsson et $a .^{8}$ more recently, reported that Mn doped GaAs displays an absorption spectrum characteristic of a substitutional acceptor, with a considerable chemical shift of its ground state with respect to its effective mass position ${ }^{9}$ but with $p$-like states remaining essentially effective masslike. In addition, Janzén et al. ${ }^{10}$ have observed the Lyman spectrum of $\mathrm{GaAs}\left(\mathrm{Cu}^{2+}\right)$.

In the present paper, we report and discuss the observation of the Lyman spectra of $\mathrm{Co}^{2+}$ and $\mathrm{Cu}^{2+}$ acceptors in $\mathrm{GaAs}$ and compare them with that of $\mathrm{Mn}^{2+}$ acceptors in the same host already observed before ${ }^{7,8}$ and confirmed by us in the present study. In addition, we present and discuss such spec- 


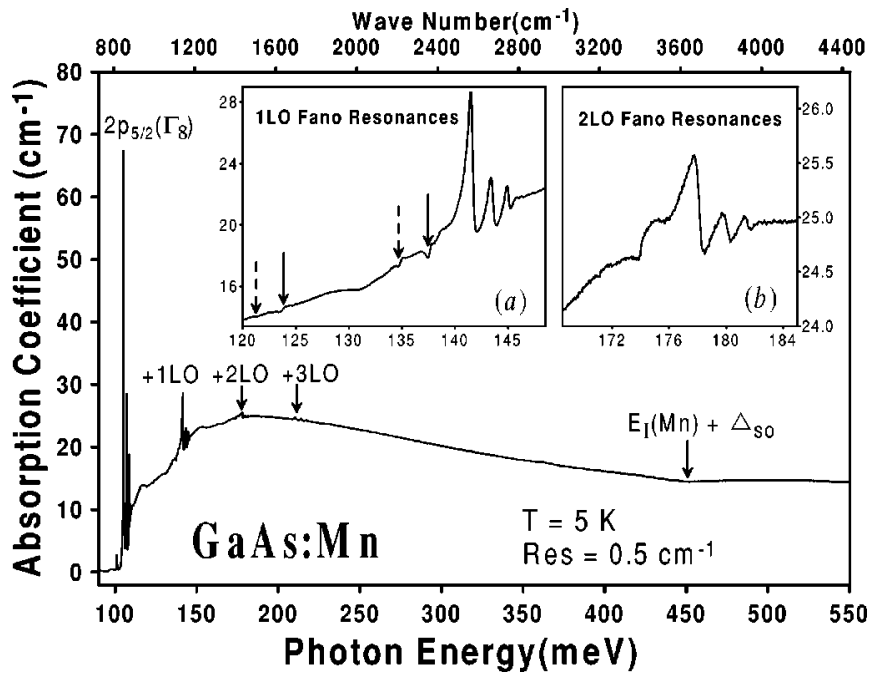

FIG. 1. Acceptor excitation (Lyman) spectrum of $\mathrm{Mn}^{2+}$ replacing $\mathrm{Ga}$ anion in GaAs. An excitation line is labeled according to its final state of the Lyman transition based on its binding energy calculated in Ref. 9. The replicas of the Lyman lines in association with zone center longitudinal optical phonon (LO) and its overtones; the onsets of photoionization to the $p_{3 / 2}$ and the $p_{1 / 2}$ valence bands; and the Fano resonances with the characteristic asymmetry in their line shapes (insets) are the other noteworthy features of the spectrum.

tra for $\operatorname{InP}\left(\mathrm{Mn}^{2+}\right)$ and $\mathrm{GaP}\left(\mathrm{Mn}^{2+}\right)$ as examples of $3 d$-TMI acceptors but in a different III-V host.

\section{EXPERIMENTAL}

The $3 d$ TMI's were introduced into nominally pure GaAs employing thermal diffusion at elevated temperatures. The relevant $3 d$ TMI of at least $4 N$ purity, uniformly deposited on the surface of the III-V specimen by evaporation in a vacuum chamber, was encapsulated in an evacuated quartz ampoule. Thermal diffusion into GaAs (InP) wafers was achieved in the presence of crushed GaAs (InP); in addition, a small quantity of the group $\mathrm{V}$ element was included to suppress sublimation. A similar procedure was adopted for $\mathrm{GaP}$.

The specimens were prepared for infrared absorption measurements by suitably polishing two approximately plane parallel surfaces, with a small wedge introduced to avoid channelling in the spectrum. The transmission spectra were recorded with a BOMEM DA.3 Fourier Transform Spectrometer, equipped with liquid nitrogen cooled $\mathrm{HgCdTe}$ and InSb detectors. ${ }^{11}$ The samples were cooled to cryogenic temperatures in a variable temperature Janis 10DT Supervaritemp optical cryostat. ${ }^{12}$

\section{EXPERIMENTAL RESULTS AND DISCUSSION}

\section{A. GaAs:Mn and GaAs:Co}

The absorption spectra of GaAs doped either with Mn or Co and recorded at temperature, $T=5 \mathrm{~K}$ are displayed in Figs. 1-3; the absorption spectrum of undoped GaAs is also included for comparison. As is evident, specimens doped

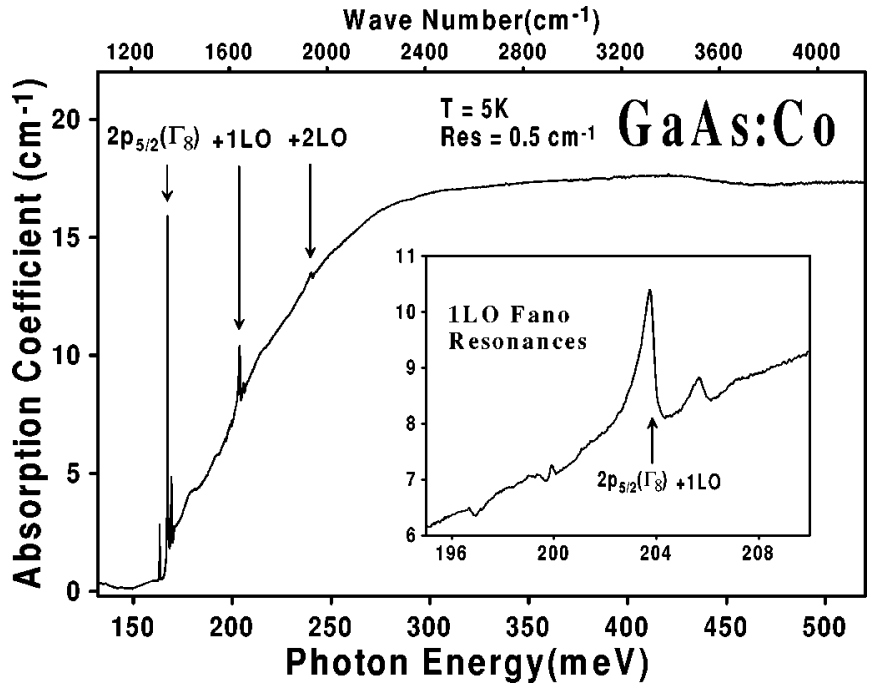

FIG. 2. The Lyman spectrum of $\mathrm{Co}^{2+}$ acceptors in GaAs along with their $1 \mathrm{LO}$ and $2 \mathrm{LO}$ replicas.

with $\mathrm{Mn}$ and Co exhibit excitation lines in the range 100$220 \mathrm{meV}$ with their positions listed in Table I.

In Fig. 1, the sharp excitation lines of the Mn acceptors in the range $100-114 \mathrm{meV}$ are followed by a photoionization continuum with its onset $\sim 112 \mathrm{meV}$, representing transitions from the ground state of the $\mathrm{Mn}$ acceptor to the $p_{3 / 2}$ valence band of GaAs; the lines, superposed on the continuum, are interpreted as the Lyman excitations in combination with one, two, and three quanta of the zone center longitudinal optical phonon (LO) of the GaAs host. ${ }^{13}$ The two insets display the $1 \mathrm{LO}$ and $2 \mathrm{LO}$ replicas of the Lyman lines on an expanded scale. In addition, two examples of TO replicas are seen in the inset (a). The line shapes of the replicas characterized by asymmetries are discussed in Sec. IV. The cross section of the photoionization continuum increases with photon energy initially, reaching a maximum and displaying a monotonic decrease beyond. For photon energies larger than the feature $\sim 452 \mathrm{meV}$ identified as " $E_{I}(\mathrm{Mn})+\triangle_{\mathrm{SO}}$," an additional contribution sets in as a consequence of excitations from the ground state of the $\mathrm{Mn}$ acceptor to the continuum of the spin-orbit split $p_{1 / 2}$ valence band. Here $E_{I}(\mathrm{Mn})$ is the ionization energy of the $\mathrm{Mn}$ acceptor and $\triangle_{\text {SO }}$, the spin-orbit splitting of the valence band. With this interpretation, along with $\triangle_{\mathrm{SO}}=341 \pm 2 \mathrm{meV}$ at $4.2 \mathrm{~K}$, ${ }^{14}$ we deduce $E_{I}(\mathrm{Mn})=111 \pm 2 \mathrm{meV}$. In the spectrum of $\mathrm{GaAs}(\mathrm{Co})$, the photoionization continuum does display a general increase in the absorption coefficient for $\hbar \omega$ $\geqslant E_{I}(\mathrm{Co})+\triangle_{\mathrm{SO}}\left[E_{I}(\mathrm{Co}) \equiv\right.$ ionization energy of Co acceptors] but the corresponding feature for Co near the onset is less distinctive in Fig. 2.

It is well known that the effective mass theory (EMT) $)^{15}$ successfully predicts the binding energies of the excited $p$-like states whose wave functions have negligible amplitudes at the Coulomb center; in contrast, the wave function of the $1 s$-like ground state has a large amplitude at the impurity site. As a consequence, the ground state is sensitive to the chemical nature of the acceptor impurity and its binding energy $E_{I}$ experiences a departure from the effective mass 


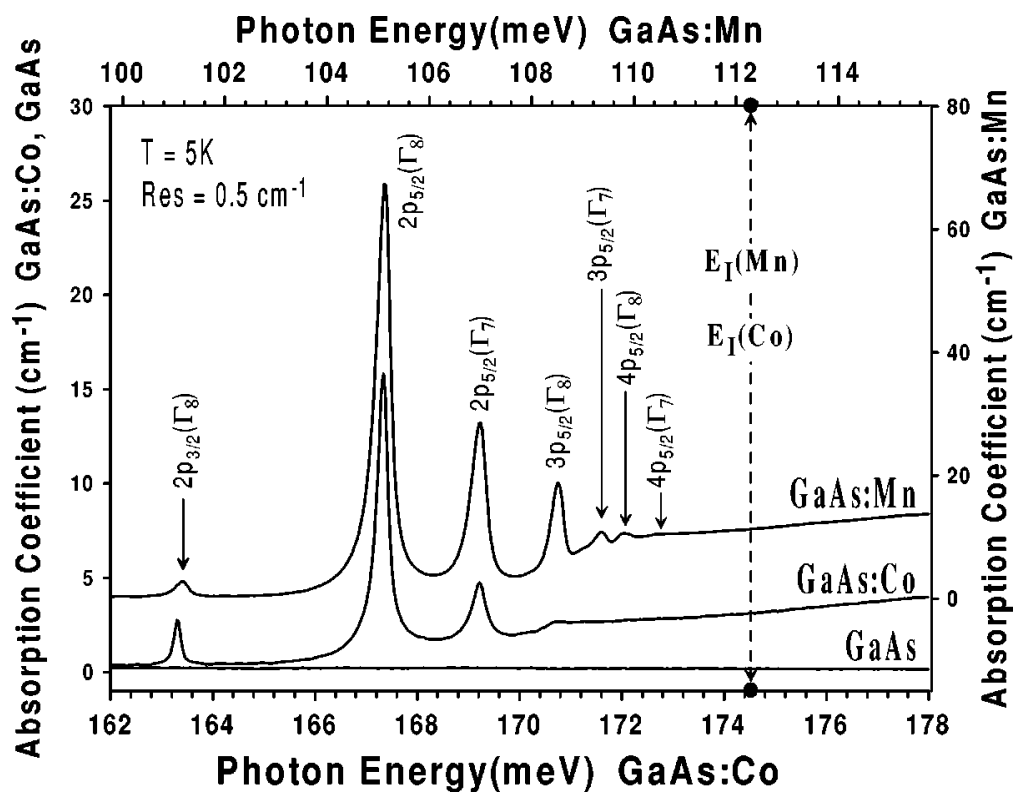

FIG. 3. The Lyman spectra of GaAs: $\mathrm{Mn}^{2+}$ and GaAs: $\mathrm{Co}^{2+}$ compared, with the $2 p_{3 / 2}\left(\Gamma_{8}\right)$ transition in each brought into coincidence. The energy scale for the two spectra being the same, the identity of the energy spacings is made evident in the figure. The binding energies of $\mathrm{Mn}$ and Co acceptors, $E_{I}\left(\mathrm{Mn}^{2+}\right)=112.32 \mathrm{meV}$ and $E_{I}\left(\mathrm{Co}^{2+}\right)=174.55 \mathrm{meV}$, are marked on their corresponding energy axis. The spectrum labeled GaAs shows the complete absence of the excitation lines in pure GaAs in the range 162-178 $\mathrm{meV}$; this is true also in the range $100-114 \mathrm{meV}$ (not shown) where the $\mathrm{Mn}^{2+}$ excitation lines occur.

value by an amount characteristic for a given impurity. The Lyman spectra for different $3 d$ TMI's thus are expected to be identical as far as the spacings of the corresponding lines and their relative intensities are concerned, but shifted in energy with respect to one another due to this chemical shift of the $s$-like ground state.

In Fig. 3, the Lyman spectra of Mn and Co acceptors in GaAs are displayed (on the same scale) with their most prominent line at $2 p_{5 / 2}\left(\Gamma_{8}\right)$ brought into coincidence. The identity of the spacings of the spectra for the two acceptor impurities is immediately evident. The spacings, in turn, are close to those predicted in the calculations of Baldereschi and Lipari $^{9}$ for single acceptors in GaAs and provide the justification for labeling the excitation lines on the basis of the final state of the transition. With this interpretation, one can add the calculated binding energy of the final state of a given transition to its experimental transition energy and thus deduce $E_{I}$. Choosing the least tightly bound final state for

TABLE I. Observed transition energies from the $1 s_{3 / 2}\left(\Gamma_{8}\right)$ ground state to $n p$ excited states of various group II and $3 d$-transition metal ions as single acceptors in $\mathrm{GaAs}$ (in $\mathrm{meV}$ ).

\begin{tabular}{lccccc}
\hline \hline Final state & $\begin{array}{c}\text { Zn } \\
(\text { Ref. 17) }\end{array}$ & $\begin{array}{c}\text { C } \\
(\text { Ref. 18) }\end{array}$ & Mn & Co & $\begin{array}{c}\text { Theory } \\
\text { (Ref. 9) }\end{array}$ \\
\hline $2 p_{3 / 2}\left(\Gamma_{8}\right)$ & 19.38 & 15.19 & 101.17 & 163.32 & 14.29 \\
$2 p_{5 / 2}\left(\Gamma_{8}\right)$ & 23.21 & 19.35 & 105.13 & 167.33 & 18.47 \\
$2 p_{5 / 2}\left(\Gamma_{7}\right)$ & 25.05 & 21.19 & 106.99 & 169.22 & 20.34 \\
$3 p_{5 / 2}\left(\Gamma_{8}\right)$ & & & 108.52 & 170.74 & $22.05^{\mathrm{a}}$ \\
$3 p_{5 / 2}\left(\Gamma_{7}\right)$ & & & 109.37 & & $22.90^{\mathrm{a}}$ \\
$4 p_{5 / 2}\left(\Gamma_{8}\right)$ & & & 109.82 & & \\
$4 p_{5 / 2}\left(\Gamma_{7}\right)$ & & & 110.55 & & \\
$\mathrm{LO}+2 p_{5 / 2}\left(\Gamma_{8}\right)$ & & & 137.78 & 199.90 & \\
$\mathrm{LO}+2 p_{5 / 2}\left(\Gamma_{8}\right)$ & & & 141.51 & 203.80 & \\
$\mathrm{LO}+2 p_{5 / 2}\left(\Gamma_{7}\right)$ & & & 143.40 & 205.68 & \\
$\mathrm{LO}+3 p_{5 / 2}\left(\Gamma_{8}\right)$ & & & 144.94 & 207.12 & \\
\hline
\end{tabular}

${ }^{\text {a See Ref. } 8 .}$ which Baldereschi and Lipari ${ }^{9}$ calculated the binding energy, $2 p_{5 / 2}\left(\Gamma_{7}\right)$, for example, we deduce $E_{I}$ by adding this binding energy viz., $5.33 \mathrm{meV}$, to the energy of the $1 s_{3 / 2}\left(\Gamma_{8}\right)$ $\rightarrow 2 p_{5 / 2}\left(\Gamma_{7}\right)$ transition in $\operatorname{GaAs}(\mathrm{Mn}, \mathrm{Co})$. Thus, the calculated ionization energies for the $\mathrm{Mn}$ and Co acceptors in GaAs are 112.32 and $174.55 \mathrm{meV}$, respectively. The value of $E_{I}$ for Mn deduced from the $E_{I}(\mathrm{Mn})+\triangle_{\mathrm{SO}}$ feature in the photoionization spectrum is consistent with this more precise value.

The assignments for the excitation lines for the $\mathrm{Mn}^{2+}$ acceptors labeled as $3 p_{5 / 2}\left(\Gamma_{8}\right)$ and $3 p_{5 / 2}\left(\Gamma_{7}\right)$ were proposed by Linnarsson et al. ${ }^{8}$ on the basis of the binding energies of the final states calculated by Said et al. ${ }^{16}$ using the parameters $\mu=\left(6 \gamma_{3}+4 \gamma_{2}\right) / 5 \gamma_{1}$ and $\delta=\left(\gamma_{3}-\gamma_{2}\right) / \gamma_{1}$, where the Luttinger parameters for GaAs are $7.65,2.41$, and 3.28 respectively. The additional lines $4 p_{5 / 2}\left(\Gamma_{8}\right)$ and $4 p_{5 / 2}\left(\Gamma_{7}\right)$ observed in the present study are similarly labeled.

In Tables I and II, the transition energies and the binding energies of the final states of the Lyman lines of the Mn and Co acceptors are compared with the theoretical calculations of Baldereschi and Lipari ${ }^{9}$ and with those of the shallow acceptors $\mathrm{Zn}$ and $\mathrm{C}$ reported in the literature. ${ }^{18,17}$

\section{B. GaP:Mn and InP:Mn}

The valence bands of the III-V's are qualitatively similar but differ in terms of their Luttinger parameters $\gamma_{1}, \gamma_{2}$, and $\gamma_{3}$, the magnitude of the spin-orbit splitting $\Delta$, and the dielectric constants $\left(\epsilon_{0}\right.$ and $\left.\epsilon_{\infty}\right) .{ }^{19}$ In order to delineate fully the role of the substitutional $3 d$ TMI's as acceptors in the III-V's, it is of interest to investigate their Lyman spectra in other hosts besides GaAs. Figures 4 and 5 show the Lyman spectra of $\mathrm{Mn}$ in $\mathrm{GaP}$ and InP, introduced by thermal diffusion.

Adopting the procedure followed for GaAs:Mn and GaAs:Co, the most prominent line in the Lyman spectrum of GaP:Mn is assigned to $2 p_{5 / 2}\left(\Gamma_{8}\right)$. Baldereschi and Lipari ${ }^{9,20}$ compute a value for the binding energy of the final state of 
TABLE II. Binding energies (in meV) of the final states of the observed transitions of various group II and $3 d$-transition metal ions as single acceptors and of $\mathrm{Cu}$ in $\mathrm{GaAs}$.

\begin{tabular}{lccccccc}
\hline \hline Final State & Zn (Ref. 17) & $\mathrm{C}($ Ref. 18) & $\mathrm{Mn}$ & $\mathrm{Co}$ & $\mathrm{Cu}(\mathrm{i})$ & $\mathrm{Cu}(\mathrm{ii})$ & Theory (Ref. 9) \\
\hline $1 s_{3 / 2}\left(\Gamma_{8}\right)$ & 30.38 & 26.52 & 112.32 & 174.55 & 157.78 & 158.61 & 25.67 \\
$2 p_{3 / 2}\left(\Gamma_{8}\right)$ & 11.00 & 11.33 & 11.15 & 11.23 & 11.28 & 11.25 & 11.38 \\
$2 p_{5 / 2}\left(\Gamma_{8}\right)$ & 7.17 & 7.17 & 7.19 & 7.22 & 7.27 & 7.24 & 7.20 \\
$2 p_{5 / 2}\left(\Gamma_{7}\right)$ & 5.33 & 5.33 & 5.33 & 5.33 & 5.33 & 5.33 & 5.33 \\
$3 p_{5 / 2}\left(\Gamma_{8}\right)$ & & & 3.80 & 3.81 & 3.06 & & $3.62^{\mathrm{a}}$ \\
$3 p_{5 / 2}\left(\Gamma_{7}\right)$ & & & 2.95 & & & & $2.77^{\mathrm{a}}$ \\
$4 p_{5 / 2}\left(\Gamma_{8}\right)$ & & 2.50 & & & & \\
$4 p_{5 / 2}\left(\Gamma_{7}\right)$ & & 1.77 & & & & \\
\hline \hline
\end{tabular}

${ }^{\mathrm{a} S e e}$ Ref. 8.

this transition to be $13.04 \mathrm{meV}$ on the basis of $\gamma_{1}=4.20$, $\gamma_{2}=0.98, \gamma_{3}=1.66$, and $\epsilon_{0}=10.75$, resulting in an ionization energy of $(387.75 \pm 0.3) \mathrm{meV}$, the error bar being estimated from the measurements on eight separate specimens. The binding energies of the final states of the transitions $2 p_{3 / 2}\left(\Gamma_{8}\right)$ and $2 p_{5 / 2}\left(\Gamma_{7}\right)$ are thus $(18.91 \pm 0.17) \mathrm{meV}$ and $(8.23 \pm 0.17) \mathrm{meV}$, respectively (see Table III). The relatively large error bars could well be a consequence of the high diffusion temperatures $\left(\sim 1000^{\circ} \mathrm{C}\right)$ over extended periods $(\sim 100 \mathrm{~h})$ followed by a rapid quench; this somewhat drastic procedure could inadvertently generate defects which produce uncontrolled strains.

The Lyman lines of InP:Mn are somewhat improved with respect to their linewidths, presumably a consequence of the somewhat lower temperatures and shorter durations for the diffusion. As in the case of GaP:Mn, we attribute the final state of the strongest line to $2 p_{5 / 2}\left(\Gamma_{8}\right)$ with the calculated binding energy of $9.98 \mathrm{meV},{ }^{20}$ yielding in turn an ionization energy of $(220.04 \pm 0.05) \mathrm{meV}^{21}$ The binding energies of the final states of the lines $2 p_{3 / 2}\left(\Gamma_{8}\right)$ and $2 p_{5 / 2}\left(\Gamma_{7}\right)$, are then deduced as $(15.38 \pm 0.11) \mathrm{meV}$ and $(6.92 \pm 0.08) \mathrm{meV}$, respectively.

It should be emphasized that the Baldereschi and Lipari values for the binding energies of $2 p_{3 / 2}\left(\Gamma_{8}\right)$ and $2 p_{5 / 2}\left(\Gamma_{7}\right)$ are 19.77 and $9.42 \mathrm{meV}$ in the case of GaP; they thus differ from the corresponding experimental values of 18.91 and $8.23 \mathrm{meV}$. For InP the theoretical values are 15.89 and 7.32 $\mathrm{meV}$ in better agreement with the experimental values of 15.38 and $6.92 \mathrm{meV}$. The differences are outside the experimental error bars, larger in $\mathrm{GaP}$ than in InP; it is premature to conclude whether they are due to the choice of the parameters used in the calculations or the quality of the host material. However, the assignments for the final states and the ionization energies appear to be acceptable.

\section{Photoionization continuum}

As discussed in the earlier sections, Figs. 1, 2, 4, and 5 display an unmistakable onset of an absorption continuum at $\hbar \omega=E_{I}$, corresponding to the photoionization of the holes bound to the $3 d$ TMI's. The physical processes underlying the photoionization spectrum involve the oscillator strength for the transitions from the $1 s$ initial state to final states in the heavy hole and light hole $p_{3 / 2}$ valence bands and their density of states. In our discussion of the experimental results on the "bound-to-continuum," we follow Edwards and Fowler. ${ }^{22}$ Their approach involves a scaled hydrogenic ground state, suitably modified to take account of central cell

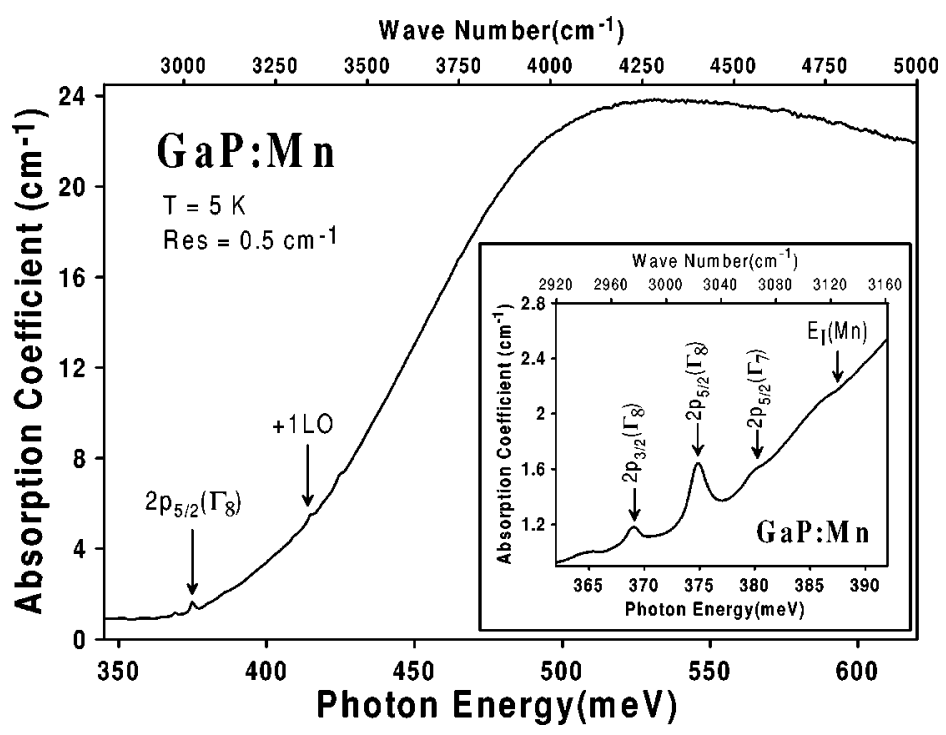

FIG. 4. Absorption spectrum of $\mathrm{Mn}^{2+}$ acceptors in GaP. Lyman transitions are followed by strong photoionization. The $2 p_{3 / 2}\left(\Gamma_{8}\right)$ transition and its 1 LO phonon replica are indicated by vertical arrows. The inset highlights the no-phonon Lyman transitions, labeled as in Fig. 3. 


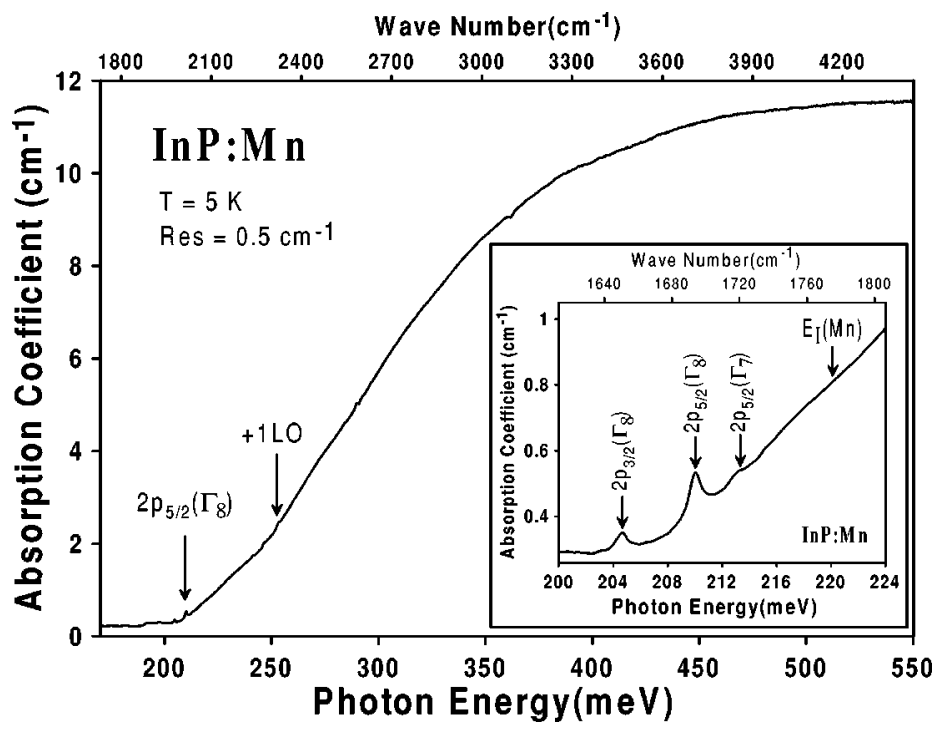

FIG. 5. Absorption spectrum of $\mathrm{Mn}^{2+}$ acceptors in InP. The $2 p_{3 / 2}\left(\Gamma_{8}\right)$ Lyman transition and its 1 LO phonon replica are indicated by arrows. The inset shows the observed Lyman transitions and $E_{I}\left(\mathrm{Mn}^{2+}\right)$, the ionization energy of $\mathrm{Mn}$ in InP. corrections and effective mass continuum states. Edwards and Fowler give two expressions for the optical absorption cross-section which are indistinguishable when compared with experimental results; we select the physically simpler dipole approximation with fewer parameters viz. Eq. (1) in Edwards and Fowler. The absorption coefficient is then given as

$$
\begin{aligned}
\alpha_{D}= & N \sigma_{D} \\
= & N\left(\frac{\mathcal{E}_{e}}{\mathcal{E}_{0}}\right)^{2} \frac{2^{9} \pi^{2} \alpha \hbar \omega m_{2}^{*}\left(Z_{2}^{\prime}\right)^{-4}}{3 \hbar^{2} \epsilon_{\infty}^{1 / 2}} \frac{\eta^{3}(1-2 \eta)^{2}(x+1)}{\left(x+\eta^{2}\right)^{6}} \\
& \times \frac{\exp \left[-4 x^{-1 / 2} \cot ^{-1}\left(\eta x^{-1 / 2}\right)\right]}{1-\exp \left(-2 \pi x^{-1 / 2}\right)}
\end{aligned}
$$

where $N$ is neutral acceptor concentration, $\left(\mathcal{E}_{e} / \mathcal{E}_{0}\right)$ is the ratio of the electric field at the defect site to the average field in the medium, ${ }^{23} \hbar \omega$, is the photon energy, and $\alpha$ is the fine-structure constant $e^{2} / \hbar c . Z_{2}^{\prime}$ is the ratio of the absolute value of the impurity charge $Z_{2}$ ( +1 for single acceptors) to the Bohr radius $a_{2}$ associated with the hydrogenic Hamiltonian from which the continuum states are obtained:

$$
Z_{2}^{\prime}=Z_{2} / a_{2}=Z_{2} m_{2}^{*} e^{2} / \hbar^{2} \epsilon_{\infty},
$$

where $m_{2}^{*}$ is the absolute value of the effective mass associated with the continuum states. $\epsilon_{\infty}$ is the optical dielectric

TABLE III. Binding energies for the energy levels of Mn acceptors in $\mathrm{GaP}$ and $\mathrm{InP}$ (in $\mathrm{meV}$ ).

\begin{tabular}{lcccc}
\hline \hline & \multicolumn{2}{c}{ GaP:Mn } & \multicolumn{2}{c}{ InP:Mn } \\
Final state & Experiment & $\begin{array}{c}\text { Theory } \\
\text { (Ref. 9) }\end{array}$ & Experiment & $\begin{array}{c}\text { Theory } \\
\text { (Ref. 9) }\end{array}$ \\
\hline $1 s_{3 / 2}\left(\Gamma_{8}\right)$ & $387.75 \pm 0.28$ & & $220.04 \pm 0.05$ & \\
$2 p_{3 / 2}\left(\Gamma_{8}\right)$ & $18.91 \pm 0.17$ & 19.77 & $15.38 \pm 0.11$ & 15.89 \\
$2 p_{5 / 2}\left(\Gamma_{8}\right)$ & 13.04 & 13.04 & 9.98 & 9.98 \\
$2 p_{5 / 2}\left(\Gamma_{7}\right)$ & $8.23 \pm 0.17$ & 9.42 & $6.92 \pm 0.08$ & 7.32 \\
\hline \hline
\end{tabular}

constant and $x$, a dimensionless energy parameter proportional to the energy $E$ measured from the threshold of the continuum absorption, is given by

$$
x=2 m_{2}^{*} E / \hbar^{2}\left(Z_{2}^{\prime}\right)^{2}
$$

The quantity $\eta$ in Eq. (1) is the ratio of the "inner" to the "outer" effective charge

$$
\eta=Z_{1}^{\prime} / Z_{2}^{\prime}
$$

where $Z_{1}^{\prime}=Z_{1} m_{1}^{*} e^{2} / \hbar^{2} \epsilon_{\infty}$ is defined with respect to the hydrogenic Hamiltonian leading to the ground state.

The fits to the experimental results are displayed in Figs. 6(a)-6(d) based on the parameters shown in Table IV and the ionization energies deduced from the Lyman spectra in Tables II and III. The effective mass parameter $m_{2}^{*}$, associated with the valence band continuum, must be the same for different acceptors in the same host. As can be seen, $m_{2}^{*}$ is indeed the same for $\mathrm{Mn}^{2+}$ and $\mathrm{Co}^{2+}$ acceptors in GaAs, being $0.152 m_{e}$. This value differs from $0.53 m_{e}$, the "density-of-state average" of the heavy and light hole band effective masses. This feature was noted by Edwards and Fowler in their analysis of group III acceptors in Si host, presumably indicative of the dominance of the light hole band and the $p_{1 / 2}$ band. In GaAs, the $p_{1 / 2}$ band maximum being separated from that of the $p_{3 / 2}$ band by $341 \mathrm{meV}$, one indeed can ignore its contribution in the range $\hbar \omega<E_{I}$ $+\Delta_{\mathrm{SO}}$. The effective charge ratio $\eta$ is 3.95 for $\mathrm{Mn}^{2+}$ whereas for $\mathrm{Co}^{2+}$, it is 5.2; the significantly larger chemical shift for $\mathrm{Co}^{2+}$ appears to be the underlying cause for its larger value of $\eta$. We note that the maximum in the photoionization spectrum occurs $68 \mathrm{meV}$ higher than the ionization energy for $\mathrm{Mn}^{2+}$ acceptors (in GaAs) whereas for $\mathrm{Co}^{2+}$ acceptors, it is $\sim 175 \mathrm{meV}$ higher. This larger separation can be ascribed to the smaller overlap of the continuum wave 

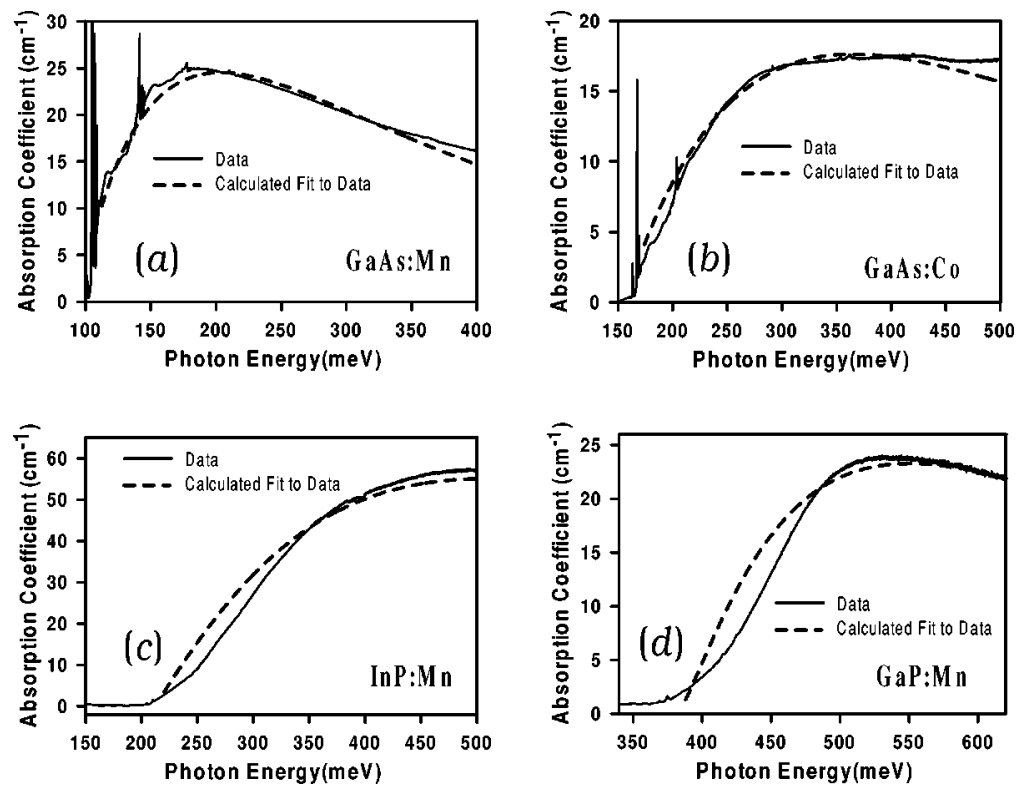

FIG. 6. $\mathrm{Mn}^{2+}$ photoionization spectrum in (a) GaAs, (c) InP, and (d) GaP. The $\mathrm{Co}^{2+}$ photoionization spectrum in GaAs is shown in (b). Solid lines indicate data while dashes show the curves computed following Edwards and Fowler (Ref. 22).

functions with the more localized wave functions of the deeper ground state, as pointed out by Edwards and Fowler ${ }^{22}$ for $\mathrm{Si}(\mathrm{Ga})$ vs $\mathrm{Si}(\mathrm{B})$.

The analysis of the photoionization spectra in terms of Eq. (1) also yields $N\left(\mathcal{E}_{e} / \mathcal{E}_{0}\right)^{2}$. The effective field ratio $\left(\mathcal{E}_{e} / \mathcal{E}_{0}\right)$ lies between ${ }^{23} \quad 1<\left(\mathcal{E}_{e} / \mathcal{E}_{0}\right)<\left[1+\left(\epsilon_{\infty}-1\right) / 3\right]$ i.e., 1 $<\left(\mathcal{E}_{e} / \mathcal{E}_{0}\right)<4.2$ for $\mathrm{GaAs} ;\left(\mathcal{E}_{e} / \mathcal{E}_{0}\right) \sim 1$ for shallow acceptors and close to the upper limit for the deeper acceptors. ${ }^{23}$ Thus the acceptor concentration for GaAs: $\mathrm{Mn}^{2+}$ lies between 5 $\times 10^{15}$ to $8.8 \times 10^{16} \mathrm{~cm}^{-3}$ for the specimen used in the results shown in Fig. 6(a). A careful analysis of Hall data can thus result in a more definitive value for $\left(\mathcal{E}_{e} / \mathcal{E}_{0}\right)$.

The curve fitting for $\mathrm{Mn}$ in $\mathrm{InP}$ and $\mathrm{GaP}$ yields $m_{2}^{*}$ as $0.05 m_{e}$ and $0.015 m_{e}$, respectively. The small magnitude of $m_{2}^{*}$ for the same impurity in these hosts is also attributed to the larger chemical shifts, i.e., for deeper impurities, the more localized ground states give larger transition matrix elements to the light-hole band. The neutral $\mathrm{Mn}^{2+}$ acceptor concentration in $\mathrm{GaP}$ is estimated to be in the range from 6 $\times 10^{15}$ to $7.7 \times 10^{15} \mathrm{~cm}^{-3}$ while it is in the range $9.5 \times 10^{15}$ to $1.42 \times 10^{17} \mathrm{~cm}^{-3}$ in InP.

The effective charge ratio $\eta$ obtained from curve fitting is 14 for GaP:Mn and 9.3 for InP:Mn. The larger values of $\eta$ for deeper impurities are also expected as a consequence of the tightly bound, more localized ground states.

\section{IV. "FANO" INTERACTION AND LINE SHAPES OF LO-PHONON ASSISTED LYMAN TRANSITIONS}

As originally recognized and formulated by Breit and Wigner $^{24}$ and by Fano, ${ }^{25}$ in the context of nuclear transitions and atomic spectroscopy, respectively, the energy coincidence of a discrete excitation with those having a continuous range, leads to striking asymmetric line shapes characterized by resonance and antiresonance features. A large number of such situations in diverse physical systems, including semiconductors, have been reported in the literature. We cite Watkins and Fowler ${ }^{26}$ and Piao et al. ${ }^{27}$ as illustrative examples particularly relevant in the discussion on the simultaneous excitation of a Lyman transition in combination with $\mathrm{n}$ quanta of zone center optical phonons $(n=1,2,3, \ldots)$. The specific case in the present investigation is the $1 \mathrm{LO}, 2 \mathrm{LO}$, and $3 \mathrm{LO}$ replicas of the Lyman excitations of Mn acceptors in GaAs, displayed in Fig. 1. The polar character of GaAs clearly favors the special role of the zone center longitudinal optic phonon (LO) and its overtones, the microscopic mechanism for the coupling being the Fröhlich interaction. ${ }^{28}$ In the photoionization spectrum shown in inset (a), the features at 137.5 and $123.8 \mathrm{meV}$ are associated with the $2 p_{3 / 2}\left(\Gamma_{8}\right)$ and the "forbidden" $2 s_{3 / 2}\left(\Gamma_{8}\right)$ Lyman transitions, respectively, involving one LO phonon (solid arrows). The accompanying set of transitions $2.7 \mathrm{meV}$ lower in energy are due to one TO

TABLE IV. Results of curve-fitting analysis of the photoionization continuum of $\mathrm{Mn}$ and Co acceptors in GaAs and $\mathrm{Mn}$ acceptors in $\mathrm{GaP}$ and InP using Eq. (1).

\begin{tabular}{lcccc}
\hline \hline Parameter & GaAs:Mn & GaAs:Co & InP:Mn & GaP:Mn \\
\hline$E_{I}(\mathrm{meV})$ & 112.32 & 174.55 & 220.04 & 387.75 \\
$m_{2}^{*} / m_{e}$ & 0.152 & 0.152 & 0.05 & 0.015 \\
$\eta$ & 3.95 & 5.2 & 9.3 & 14 \\
$N\left(\mathcal{E}_{e} / \mathcal{E}_{0}\right)^{2}\left(\mathrm{~cm}^{-3}\right)$ & $8.82 \times 10^{16}$ & $1.15 \times 10^{17}$ & $1.42 \times 10^{17}$ & $7.7 \times 10^{15}$ \\
\hline \hline
\end{tabular}


phonon assisted Fano resonances of the same Lyman lines (dashed arrows). The latter are presumably mediated through deformation potential interactions. These features appear in the photothermal ionization spectra reported in Ref. 8. The Breit-Wigner-Fano resonance interaction in semiconductors is one of the most interesting example of electron-phonon interactions. ${ }^{29,30}$

The analysis of the "LO+Lyman" transitions in terms of their characteristic features-asymmetry, resonance peak and antiresonance dip, spectral width, and shifts with respect to their unperturbed positions-is carried out on the basis of Fano's formulation. We denote the acceptor ground state by the symbol $|0\rangle$ and the excited state associated with a particular absorption line by $|f n\rangle$ where $f$ designates the electronic state of the excitation and $n=1,2, \ldots$, the number of optical phonons associated with the line in question. In contrast to $|0\rangle \rightarrow|f 0\rangle$, the zero-phonon excitation in the Lyman spectrum, the phonon-assisted transitions $|0\rangle \rightarrow|f n\rangle$ ( $n$ $=1,2, \ldots)$, are resonant with the valence band. We describe the valence band states by $|\mathbf{k}\rangle$ where this symbol includes not only the continuous wave vector $\mathbf{k}$, but also a band index (light and heavy hole bands) which we need not specify explicitly. The states having energies close to $E_{f n}=E_{f}+n \hbar \omega$ form a manifold which can be described by an interaction Hamiltonian $V$ whose matrix elements are

$$
\begin{gathered}
\langle f n|V| f n\rangle=E_{f n}, \\
\left\langle\mathbf{k}^{\prime}|V| \mathbf{k}\right\rangle=E(\mathbf{k}) \delta_{k^{\prime} k},
\end{gathered}
$$

and

$$
\langle\mathbf{k}|V| f n\rangle=V_{k} .
$$

The coupling between $|f n\rangle$ and the continuum can be described by a wave function of the form

$$
\Psi=a|f n\rangle+\sum_{k} b_{k}|\mathbf{k}\rangle
$$

which we assume to be properly normalized according to $\delta$-function normalization. From the Schrödinger equation $H \Psi=E \Psi$, we obtain

$$
\left(E-E_{f n}\right) a=\sum_{k} b_{k} V_{k}^{*}
$$

and

$$
a V_{k}=b_{k}[E-E(\mathbf{k})] .
$$

Since the set $\{|\mathbf{k}\rangle\}$ forms a continuum, the singularity in $b_{k}$ as $[E-E(\mathbf{k})]$ passes through zero, can be avoided by taking the integral over $\mathbf{k}$ (here denoted with a summation symbol for convenience) as a principal part involving the denominator plus a $\delta$ function. Following Fano, we write

$$
b_{k}=a\left[\frac{V_{k}}{E-E(\mathbf{k})}+z(\mathbf{k}) \delta[E-E(\mathbf{k})] V_{k}\right] .
$$

The dimensionless quantity $z(\mathbf{k})$ is obtained substituting $b_{k}$ in Eq. (9) and cancelling $a$,

$$
z(\mathbf{k})=\frac{E-E_{f n}-F(E)}{\left|V_{k}\right|^{2}}
$$

where

$$
F(E)=\mathcal{P} \sum_{k} \frac{\left|V_{k}\right|^{2}}{E-E(\mathbf{k})} .
$$

The symbol $\mathcal{P}$ in Eqs. (11) and (13) indicates that, when integrating over $\mathbf{k}$, one must take the principal part of the integral.

The probability per unit time for an optical transition to $\Psi$ to occur is proportional to $\left|\left\langle\Psi\left|H^{\prime}\right| 0\right\rangle\right|^{2}$ where $H^{\prime}$ is the interaction of the electronic system with the electromagnetic field of the incident radiation. The quantity $|a|^{2}$, obtained by $\delta$-function normalization is given in Ref. 25 [Eqs. (10)-(13) and Appendix A]. We write

$$
|a|=\left(1+\epsilon^{2}\right)^{-1 / 2}\left(\pi\left|V_{k}\right|\right)^{-1},
$$

where

$$
\epsilon=\frac{E-E_{f n}-F(E)}{\pi\left|V_{k}\right|^{2}}=\frac{E-E_{f n}-F(E)}{(\Gamma / 2)}
$$

with $\Gamma=2 \pi\left|V_{k}\right|^{2}$, the spectral width of $|f n\rangle$ modified by mixture with the continuum states. The matrix element $\left\langle\Psi\left|H^{\prime}\right| 0\right\rangle$ is

$$
\begin{aligned}
\left\langle\Psi\left|H^{\prime}\right| 0\right\rangle= & a^{*}\left(\left\langle f n\left|H^{\prime}\right| 0\right\rangle+\mathcal{P} \sum_{k} \frac{\langle f n|V| \mathbf{k}\rangle\left\langle\mathbf{k}\left|H^{\prime}\right| 0\right\rangle}{E-E(\mathbf{k})}\right. \\
& \left.+z(\mathbf{k})\langle f n|V| \mathbf{k}\rangle\left\langle\mathbf{k}\left|H^{\prime}\right| 0\right\rangle\right)
\end{aligned}
$$

A quantity $q$ is defined [see Ref. (25)] by

$$
\begin{aligned}
q= & \left(\left\langle f n\left|H^{\prime}\right| 0\right\rangle+\mathcal{P} \sum_{k} \frac{\langle f n|V| \mathbf{k}\rangle\left\langle\mathbf{k}\left|H^{\prime}\right| 0\right\rangle}{E-E(\mathbf{k})}\right) \\
& \times\left(\pi\langle f n|V| \mathbf{k}\rangle\left\langle\mathbf{k}\left|H^{\prime}\right| 0\right\rangle\right)^{-1} .
\end{aligned}
$$

Noting $z(\mathbf{k})=\pi \epsilon$,

$$
\frac{\left\langle\Psi\left|H^{\prime}\right| 0\right\rangle}{\left\langle\mathbf{k}\left|H^{\prime}\right| 0\right\rangle}=\frac{(q+\epsilon)}{\left(1+\epsilon^{2}\right)^{1 / 2}} .
$$

Since the phase factors in the definition of $q$ cancel out, this quantity is real. Thus,

$$
\left|\frac{\left\langle\Psi\left|H^{\prime}\right| 0\right\rangle}{\left\langle\mathbf{k}\left|H^{\prime}\right| 0\right\rangle}\right|^{2}=\frac{(q+\epsilon)^{2}}{1+\epsilon^{2}}=1+\frac{q^{2}+2 \epsilon q-1}{1+\epsilon^{2}} .
$$

Lawætz, ${ }^{30}$ following a concise derivation of the above concepts, points out the existence of channels other than those leading to the Fano interaction and a part of the continuum not contributing to it. Thus Eq. (19) should be rewritten in terms of the total absorption $I$ from which $I_{b}$, the background absorption due to the channels not contributing to Fano interaction, has been subtracted, and normalized to 

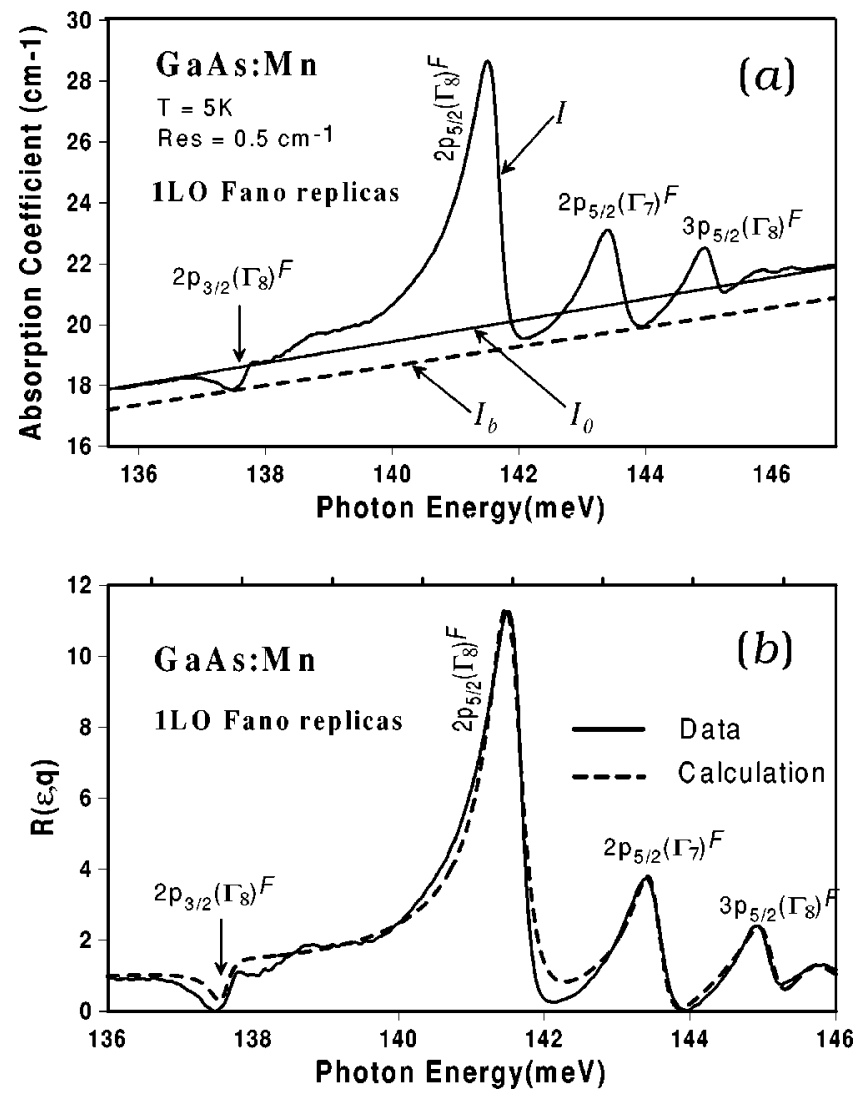

FIG. 7. Fano line-shape analysis of $1 \mathrm{LO}$ phonon resonances. (a) 1 LO Fano replicas, $2 p_{3 / 2}\left(\Gamma_{8}\right)^{F} \cdots 3 p_{5 / 2}\left(\Gamma_{8}\right)^{F}$, are seen as peaks and dips. $I$ represents the total intensity while $I_{b}$ is the background due to channels not contributing to Fano interaction and $I_{0}$ is the total absorption in the absence Fano resonance (see text). (b) Solid curve shows the data processed according to Eq. (20) and dashed curve shows the calculated fit using the parameters in Table V.

$I_{0}-I_{b}$; here $I_{0}$ is the intensity of the total absorbed light if there were no resonant interaction. Hence,

$$
R(\epsilon, q)=\frac{I-I_{b}}{I_{0}-I_{b}}=\frac{(q+\epsilon)^{2}}{1+\epsilon^{2}} .
$$

In Fig. 7(a), we display the 1LO phonon replicas of the Lyman spectrum of GaAs:Mn, where $I, I_{b}$, and $I_{0}$ are identified. In Fig. 7(b), the spectrum, processed according to Eq. (20), is presented along with the theoretical fits for the LOphonon replicas of the first four Lyman transitions, viz., $2 p_{3 / 2}\left(\Gamma_{8}\right)^{F}, 2 p_{5 / 2}\left(\Gamma_{8}\right)^{F}, 2 p_{5 / 2}\left(\Gamma_{7}\right)^{F}$, and $3 p_{5 / 2}\left(\Gamma_{8}\right)^{F}$. Fol-

TABLE V. Parameters for the 1 LO Fano replicas of the first four Lyman transitions of acceptor bound holes in GaAs:Mn, all units being in $\mathrm{meV}$.

\begin{tabular}{lccccc}
\hline \hline 1 LO replica & $E_{f}+\hbar \omega_{\mathrm{LO}}$ & $E_{0}$ & $F$ & $q$ & $\Gamma$ \\
\hline $2 p_{3 / 2}\left(\Gamma_{8}\right)^{F}$ & 137.618 & 137.59 & -0.03 & 0.33 & 0.3 \\
$2 p_{5 / 2}\left(\Gamma_{8}\right)^{F}$ & 141.578 & 141.56 & -0.018 & -3.16 & 0.57 \\
$2 p_{5 / 2}\left(\Gamma_{7}\right)^{F}$ & 143.436 & 143.54 & 0.1 & -1.76 & 0.45 \\
$3 p_{5 / 2}\left(\Gamma_{8}\right)^{F}$ & 144.964 & 145.10 & 0.14 & -1.18 & 0.465 \\
\hline \hline
\end{tabular}

TABLE VI. Parameters for the 2 LO Fano replicas of the first four Lyman transitions of acceptor bound holes in GaAs:Mn, all units being in $\mathrm{meV}$.

\begin{tabular}{lcccc}
\hline \hline 2 LO replica & $E_{f}+2 \hbar \omega_{\mathrm{LO}}$ & $F$ & $q$ & $\Gamma$ \\
\hline $2 p_{3 / 2}\left(\Gamma_{8}\right)^{F F}$ & 174.054 & -0.08 & 0.7 & 0.54 \\
$2 p_{5 / 2}\left(\Gamma_{8}\right)^{F F}$ & 178.034 & -0.04 & -2.05 & 1.0 \\
$2 p_{5 / 2}\left(\Gamma_{7}\right)^{F F}$ & 179.884 & 0.1 & -1.15 & 0.6 \\
$3 p_{5 / 2}\left(\Gamma_{8}\right)^{F F}$ & 181.414 & 0.15 & -1.0 & 0.7 \\
\hline \hline
\end{tabular}

lowing Piao et al. ${ }^{27}$ one can obtain for each transition, the values of $q, \Gamma$, and $F$ from the values of $\epsilon$ and $R$ given by $R_{\max }=q^{2}+1$ for $\epsilon_{\max }=q^{-1} ; R_{\min }=0$ for $\epsilon_{\min }=-q$; and $R_{0}=q^{2}$ for $E=E_{0}$ with $F=E_{0}-\left(E_{f}+\hbar \omega_{\mathrm{LO}}\right)$ defined by $\epsilon$ $=0$. Also, $\Gamma$ follows from $\epsilon_{\max }-\epsilon_{\min }=q+q^{-1}=2\left(\hbar \omega_{\max }\right.$ $\left.-\hbar \omega_{\min }\right) / \Gamma$. These parameters are tabulated in Tables $\mathrm{V}$ and VI for the $1 \mathrm{LO}$ and $2 \mathrm{LO}$ replicas, respectively.

\section{CONCLUDING REMARKS}

The present study has focused on the Lyman spectra of several $3 d$-transition metals $(\mathrm{Mn}, \mathrm{Co}, \mathrm{Cu}$ ), substitutionally incorporated in the III-V semiconductors ( $\mathrm{GaAs}, \mathrm{GaP}$, and InP) by replacing the cations. The acceptor concentration resulting from the diffusion techniques employed viz., $10^{15}$ to $10^{17} \mathrm{~cm}^{-3}$, corresponds to a dilution at which wavefunctions of the acceptor-bound holes do not overlap as testified by the sharpness of the Lyman transitions observed at the cryogenic temperatures. In particular, $\mathrm{Mn}^{2+}$ and $\mathrm{Co}^{2+}$ acceptors in GaAs yielded sharp and intense transitions terminating at excited states preceding the photoionization threshold. The onset of the photoionization is in excellent agreement with the sum of the transition energy and the

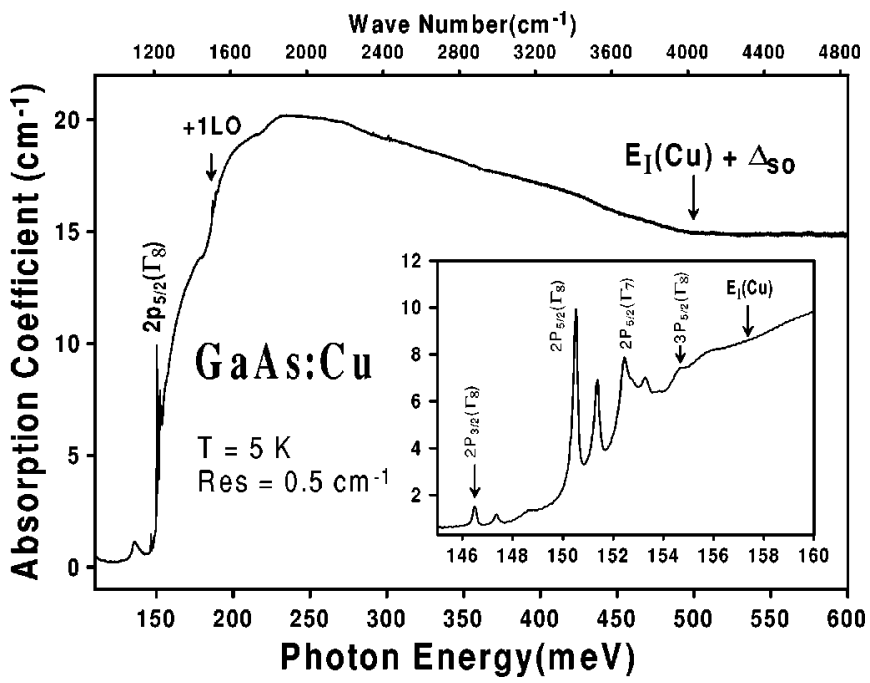

FIG. 8. The absorption spectrum of $\mathrm{Cu}$ acceptors in GaAs. Lyman lines, photoionization continuum, +1 LO Fano replicas superimposed on the photoionization continuum, and the spin-orbit split valence band absorption edge $\left[E_{I}(\mathrm{Cu})+\Delta_{\mathrm{SO}}\right]$, indicated on the figure are all characteristics of acceptor bound holes in a III-V host. The inset shows a doublet structure in each Lyman line of $\mathrm{Cu}$. 
binding energy of its final state calculated in the effective mass theory. ${ }^{9}$ While the spectra for $\mathrm{Mn}^{2+}$ acceptors in $\mathrm{GaP}$ and InP display well resolved Lyman transitions followed by photoionization, their sharpness appear to be degraded presumably because of the high diffusion temperatures. In this context, photothermal ionization spectroscopy ${ }^{31}$ allows more excitation lines to be observed, on the one hand, and explore lower acceptor concentrations, on the other, as indeed demonstrated in Linnarsson et al. ${ }^{8}$ Among the $3 d$ TMI's, $\mathrm{Cr}$ and $\mathrm{Cu}$ are exceptions in that, the atomic configurations, instead of being (i) $3 d^{4} 4 s^{2}$ and $3 d^{9} 4 s^{2}$, are (ii) $3 d^{5} 4 s^{1}$ and $3 d^{10} 4 s^{1}$, respectively. The behavior of these TMI's as acceptors will thus differ from that of the others if the $3 d$ shell does not change during incorporation by the substitution of the cation; they will then acquire one electron from the valence band in order to complete the covalent bonds in case (i), whereas in (ii) two are needed to accomplish it. We thus have a H-like single acceptor in (i) as opposed to a neutral He-like double acceptor in (ii). The binding energies of the final states for both cases will be close if the experience with $\mathrm{B}, \mathrm{Zn}$, and $\mathrm{Cu}$ in $\mathrm{Ge}$ is a guide.

In Fig. 8, we display the Lyman spectrum of $\mathrm{Cu}$ acceptors introduced into GaAs by thermal diffusion. The general appearance of the spectrum bears a striking resemblance to those of $\mathrm{Mn}^{2+}$ and $\mathrm{Co}^{2+}$ in Figs. 1-3. The binding energies for the final states listed in Table II emphasize quantitatively this correspondence. The striking feature, however, is a second series separated by $\sim 0.84 \mathrm{meV}$. Measurements as a function of temperature in the range $1.8-10 \mathrm{~K}$ showed no increase with $1 / T$ in the ratio of intensity of the higher to that of the lower energy component of a doublet. This appears to indicate the existence of two independent $\mathrm{Cu}$ centers. It is tempting to suggest that they correspond to the coexistence of He-like $3 d^{10} 4 s^{2} 4 p^{1}, \mathrm{Cu}^{2-}$ and H-like $3 d^{9} 4 s^{2} 4 p^{1}, \mathrm{Cu}^{-}$ Coulomb centers. The alternative of one of the centers being $\mathrm{Cu}$ acceptor associated with a defect is, however, not ruled out and further investigations are clearly needed.

\section{ACKNOWLEDGMENTS}

We thank the National Science Foundation (Grant Nos. DMR-0102699 and ESC-0129853) and Purdue University (Academic Reinvestment Grant) for the support of the studies reported in the present paper.
${ }^{1}$ Diluted Magnetic Semiconductors, edited by J. K. Furdyna and J. Kossut, Vol. 25 of Semiconductors and Semimetals, edited by R. K. Willardson and A. C. Beer (Academic, New York, 1988).

${ }^{2}$ H. Munekata, H. Ohno, S. von Molnar, A. Segmüller, L. L. Chang, and L. Esaki, Phys. Rev. Lett. 63, 1849 (1989).

${ }^{3}$ T. Dietl, H. Ohno, F. Matsukura, J. Cibert, and D. Ferrand, Science (Washington, DC, U.S.) 287, 1019 (2000).

${ }^{4}$ R. Fitzgerald, Phys. Today April, 21 (2000).

${ }^{5}$ P. Kacman, Semicond. Sci. Technol. 16, R25 (2001).

${ }^{6}$ H. Ohno and F. Matsukura, Solid State Commun. 117, 179 (2001).

${ }^{7}$ R. A. Chapman and W. G. Hutchinson, Phys. Rev. Lett. 18, 443 (1967).

${ }^{8}$ M. Linnarsson, E. Janzén, B. Monemar, M. Kleverman, and A. Thilderkvist, Phys. Rev. B 55, 6938 (1997).

${ }^{9}$ A. Baldereschi and N. O. Lipari, Phys. Rev. B 9, 1525 (1974).

${ }^{10}$ E. Janzén, M. Linnarson, B. Monemar, and M. Kleverman, in Impurities, Defects, and Diffusion in Semiconductors: Bulk and Layered Structures, edited by J. Bernholc, E.E. Haller, and D.J. Wolford, MRS Symposia Proceedings No. 163 (Materials Research Society, Pittsburgh, 1990).

${ }^{11}$ ABB BOMEM Inc. 585 Charest BLVD East Suite 300 Québec (Québec) G1K 9H4 Canada.

${ }^{12}$ Janis Research Company Inc., 2 Jewel Drive, Wilmington, MA 01887-0696.

${ }^{13}$ For the LO and TO frequencies of GaAs, see Table II in M. D. Sciacca, A. J. Mayur, Eunsoon Oh, A. K. Ramdas, S. Rodriquez, J. K. Furdyna, M. R. Melloch, C. P. Beetz, and W. S. Yoo, Phys. Rev. B 51, 7744 (1995).

${ }^{14}$ D. E. Aspnes and A. A. Studna, Phys. Rev. B 7, 4605 (1973).

${ }^{15}$ See, for example, A.K. Ramdas and S. Rodriguez, Rep. Prog. Phys. 44, 1297 (1981).

${ }^{16}$ M. Said, M. A. Kanehisa, M. Balkanski, and Y. Saad, Phys. Rev. B 35, 687 (1987).
${ }^{17}$ R. F. Kirkman, R. A. Stradling, and P. J. Lin-Chung, J. Phys. C 11, 419 (1978).

${ }^{18}$ R. Artzmüller, M. Dahl, J. Kraus, G. Schaack, and J. Schubert, J. Phys.: Condens. Matter 3, 6775 (1991).

${ }^{19}$ M. L. Cohen and J. R. Chelikowsky, Electronic Structure and Optical Properties of Semiconductors (Springer-Verlag, Heidelberg, 1988).

${ }^{20}$ A. Baldereschi and N. O. Lipari in Ref. 9 use $\gamma_{1}=4.20, \gamma_{2}$ $=0.98, \gamma_{3}=1.66$, and $\epsilon_{0}=10.75$ for $\mathrm{GaP}$ whereas, the corresponding values used by them in the case of InP are 6.28, 2.08, 2.76 , and 12.4 , respectively.

${ }^{21}$ B. Lambert, B. Clerjaud, C. Naud, B. Deveaud, G. Picoli, and Y. Toudic, The Thirteenth International, Conference on Defects in Semiconductors (The Metalurgical Society of AIME, Warrendale, PA, 1985), pp. 1141-1147.

${ }^{22}$ A. H. Edwards and W. B. Fowler, Phys. Rev. B 16, 3613 (1977).

${ }^{23}$ D. L. Dexter, Solid State Physics 6 (Academic Press, New York 1958), Vol. 6, p. 353.

${ }^{24}$ G. Breit and E. Wigner, Phys. Rev. 49, 519 (1936).

${ }^{25}$ U. Fano, Phys. Rev. 124, 1866 (1961).

${ }^{26}$ G. D. Watkins and W. B. Fowler, Phys. Rev. B 16, 4524 (1977).

${ }^{27}$ G. Piao, R. A. Lewis, and P. Fisher, Solid State Commun. 75, 835 (1990).

${ }^{28}$ For a particularly physical presentation, see P. Y. Yu and M. Cardona, Fundamentals of Semiconductors (Springer, Heidelberg, 1996), Chap. 3.

${ }^{29}$ See also M. V. Klein in Light Scattering in Solids I, edited by M. Cardona (Springer, Heidelberg, 1983), Chap. 4, for electronphonon coupled excitations explored with Raman spectroscopy.

${ }^{30}$ P. Lawætz, Ph.D. thesis, Technical University of Denmark, Lyngby, Denmark, 1978.

${ }^{31}$ See, for example, Sh. M. Kogan and T. M. Lifshits, Phys. Status Solidi A 39, 11 (1977). 